\title{
Properties of the cosmological filament between two clusters: A possible detection of a large-scale accretion shock by Suzaku
}

\author{
H. Akamatsu ${ }^{1}$, Y. Fujita ${ }^{2}$, T. Akahori ${ }^{3}$, Y. Ishisaki ${ }^{4}$, K. Hayashida $^{2}$, A. Hoshino ${ }^{5}$, \\ F. Mernier ${ }^{1,6}$, K. Yoshikawa ${ }^{7}$, K. Sato ${ }^{8}$, and J. S. Kaastra ${ }^{1,6}$ \\ 1 SRON Netherlands Institute for Space Research, Sorbonnelaan 2, 3584 CA Utrecht, The Netherlands \\ e-mail: h.akamatsu@sron.nl \\ 2 Department of Earth and Space Science, Graduate School of Science, Osaka University, Toyonaka, 565-0871 Osaka, Japan \\ 3 Graduate School of Science and Engineering, Kagoshima University, 1-21-35, Korimoto, Kagoshima, 890-0065 Kagoshima, Japan \\ ${ }^{4}$ Department of Physics, Tokyo Metropolitan University, 1-1 Minami-Osawa, Hachioji, 192-0397 Tokyo, Japan \\ 5 Research Center for Measurement in Advanced Science, Faculty of Science, Rikkyo University 3-34-1, Nishi-Ikebukuro, \\ Toshima-ku, 171-8501 Tokyo, Japan \\ ${ }^{6}$ Leiden Observatory, Leiden University, PO Box 9513, 2300 RA Leiden, The Netherlands \\ 7 Center for Computational Sciences, University of Tsukuba, 1-1-1 Tennodai, Tsukuba, 305-8571 Ibaraki, Japan \\ 8 Department of Physics, Tokyo University of Science, 1-3 Kagurazaka, Shinjyuku-ku, Tokyo 162-8601, Japan
}

Received 25 January 2017 / Accepted 18 April 2017

\begin{abstract}
We report on the results of a Suzaku observation of the plasma in the filament located between the two massive clusters of galaxies, Abell 399 and Abell 401. Abell $399(z=0.0724)$ and Abell $401(z=0.0737)$ are expected to be in the initial phase of a cluster merger. In the region between the two clusters, we find a clear enhancement in the temperature of the filament plasma from $4 \mathrm{keV}$ (expected value of a typical cluster temperature profile) to $k T \sim 6.5 \mathrm{keV}$. Our analysis also shows that filament plasma is present out to a radial distance of $15^{\prime}(1.3 \mathrm{Mpc})$ from a line connecting the two clusters. The temperature profile is characterized by an almost flat radial shape with $k T \sim 6-7 \mathrm{keV}$ within $10^{\prime}$ or $\sim 0.8 \mathrm{Mpc}$. Across $r=8^{\prime}$ from the axis, the temperature of the filament plasma shows a drop from $6.3 \mathrm{keV}$ to $5.1 \mathrm{keV}$, indicating the presence of a shock front. The Mach number based on the temperature drop is estimated to be $\mathcal{M} \sim 1.3$. We also successfully determined the abundance profile up to $15^{\prime}(1.3 \mathrm{Mpc})$, showing an almost constant value $(Z=0.3$ solar $)$ at the cluster outskirts. We estimated the Compton y parameter to be $\sim 14.5 \pm 1.3 \times 10^{-6}$, which is in agreement with the Planck results $\left(14-17 \times 10^{-6}\right.$ on the filament). The line-of-sight depth of the filament is $l \sim 1.1 \mathrm{Mpc}$, which indicates that the geometry of filament is likely a pancake shape rather than cylindrical. The total mass of the filamentary structure is $\sim 7.7 \times 10^{13} M_{\odot}$. We discuss a possible interpretation of the drop of X-ray emission at the rim of the filament, which was pushed out by the merging activity and formed by the accretion flow induced by the gravitational force of the filament.
\end{abstract}

Key words. X-rays: galaxies: clusters - intergalactic medium - galaxies: abundances - large-scale structure of Universe galaxies: clusters: intracluster medium

\section{Introduction}

According to the scenario of hierarchical structure formation, two important predictions are made: first, large-scale accretion shocks located between the virial and turnaround radii of clusters of galaxies and, second, about one-third of the baryons in the Universe exist as a warm-hot intergalactic medium (WHIM) in the large-scale filamentary structure of galaxies (Fukugita et al. 1998; Cen \& Ostriker 1999; Davé et al. 2001; Yoshikawa et al. 2003, 2004; Springel et al. 2005). In spite of their importance in terms of the cluster evolution and the generation of high energy photons and cosmic rays $(\mathrm{CR}$; e.g., Kang et al. 1996; Miniati et al. 2001a,b; Miniati 2003; Ryu et al. 2003; Skillman et al. 2008, and see references therein), largescale accretion shocks have not been detected yet because of limited capabilities of current observatories. Studying properties of the WHIM such as density, temperature, and metallicity is essential to understand the evolution of the an intergalactic medium (IGM) and galaxies. The properties of the WHIM in filaments of galaxies have been investigated before. For instance, the density of the WHIM in filaments has been deduced from observations of X-ray absorption lines (Takei et al. 2002; Fujimoto et al. 2004; Nicastro et al. 2005, 2013; Kaastra et al. 2006; Buote et al. 2009; Fang et al. 2010; Zappacosta et al. 2010). Up to now, however, X-ray emission from the WHIM has not been detected yet owing to the limited sensitivities of X-ray spectrometers, such as CCD cameras, except for reports of angular correlation of the diffuse X-ray background (Galeazzi et al. 2009), emission in a filament linking two clusters of galaxies (Werner et al. 2008) and a specific case (Eckert et al. 2015). The latter case, which is is slightly denser and hotter than what expected for the WHIM, would be promising to observe with present-day X-ray detectors, although such a medium might be partly mixed with an intracluster medium (ICM).

Abell 399 (hereafter A399) and Abell 401 (A401) are cluster associations for which there is known a filamentary structure that links them. We define the collision axis as the axis connecting the two cluster centers. Table 1 summarizes basic information of 
Table 1. Basic information of Abell 399 and Abell 401.

\begin{tabular}{llrrr}
\hline \hline Cluster & $(\mathrm{RA}, \mathrm{Dec})^{a}$ & $z$ & $\begin{array}{c}k T^{b} \\
\mathrm{keV}\end{array}$ & $\begin{array}{c}r_{200}{ }^{c} \\
\mathrm{Mpc}\end{array}$ \\
\hline A399 & $\left(2^{\mathrm{h}} 57^{\mathrm{m}},+13^{\mathrm{d}} 02^{\mathrm{m}}\right)$ & 0.0724 & 7.23 & 2.19 \\
A401 & $\left(2^{\mathrm{h}} 58^{\mathrm{m}},+13^{\mathrm{d}} 34^{\mathrm{m}}\right)$ & 0.0737 & 8.47 & 2.19 \\
\hline
\end{tabular}

References. ${ }^{(a)}$ Oegerle \& Hill (2001). ${ }^{(b)}$ Sakelliou \& Ponman (2004). ${ }^{(c)}$ Reiprich \& Böhringer (2002).

these clusters. Line of sight and projected distances between the centers of the clusters are $\sim 6 \mathrm{Mpc}$ and $\sim 3 \mathrm{Mpc}$, respectively, and thus the three-dimensional distance between them is $\sim 7 \mathrm{Mpc}$.

Ulmer et al. (1979) and Ulmer \& Cruddace (1981) first reported diffuse X-ray emission extending 2 Mpc between A399 and A401 using HEAO1 and HEAO2 (Einstein). Fujita et al. (1996b) observed A399 and A401 with ASCA and confirmed an excess of X-ray emission over the value expected for the case of a simple superposition of the clusters. These authors showed the presence of IGM between A399 and A401. These results were also confirmed by Sakelliou \& Ponman (2004) with XMM-Newton. Later, Fujita et al. (2008) observed a field between both clusters with Suzaku and found a metallicity of 0.2 solar (abundance table; Anders \& Grevesse 1989). They claimed that the proto-cluster region was polluted with metals by superwinds from galaxies before the clusters were formed. A similar result was also reported with Suzaku observations of the Perseus cluster (Werner et al. 2013). More recently, Planck has detected the thermal Sunyaev-Zel'ovich (tSZ) effect (Planck Collaboration VIII 2013). Combining Planck's tSZ results and ROSAT X-ray data, these authors derived a temperature and an electron density of the filament of $k T=7.1 \pm 0.9 \mathrm{keV}$, $n_{\mathrm{e}}=(3.7 \pm 0.2) \times 10^{-4} \mathrm{~cm}^{-3}$, respectively.

Akahori \& Yoshikawa (2008) performed $N$-body and smoothed particle hydrodynamics simulations of A399 and A401 to examine the effects of a non-equilibrium ionization state and an electron-ion two-temperature structure on the estimation of the metallicity in the linked region. Furthermore, these authors predicted that shock fronts could exist offset from the collision axis. Cosmological simulations suggest that the density perpendicular to the collision axis would have an exponential profile (e.g., Colberg et al. 2005; Dolag et al. 2006).

In this paper, we present the results of a reanalysis of the deep $150 \mathrm{ks}$ Suzaku observation of the region between A399 and A401. We assume cosmological parameters $H_{0}=$ $70 \mathrm{~km} \mathrm{~s}^{-1} \mathrm{Mpc}^{-1}, \Omega_{\mathrm{M}}=0.27$, and $\Omega_{\Lambda}=0.73$. As our fiducial reference for the proto-solar abundances denoted by $Z_{\odot}$, we adopt Lodders et al. (2009) and Galactic absorption with $n_{\mathrm{H}}=1.0 \times 10^{21} \mathrm{~cm}^{-2}$ (Dickey \& Lockman 1990). Unless otherwise stated, the errors shown in this paper are $68 \%$ confidence for a single parameter.

\section{Data reduction}

Suzaku performed a deep $150 \mathrm{ks}$ observation in the region between A399 and A401 during AO1 (from 2006-08-19, ObsID: 801020010). In this paper, we discuss the results based on Suzaku XIS data. The Suzaku XIS consists of three frontside illuminated (FI) CCD chips (XIS0, XIS2, and XIS3) and one back-side illuminated (BI) chip (XIS1; Koyama et al. 2007). All observations were performed with either the normal $5 \times 5$ or $3 \times 3$ clocking mode. We used FTOOLS from the HEADAS 6.12 package and CALDB 2016-06-07 for all of the Suzaku data

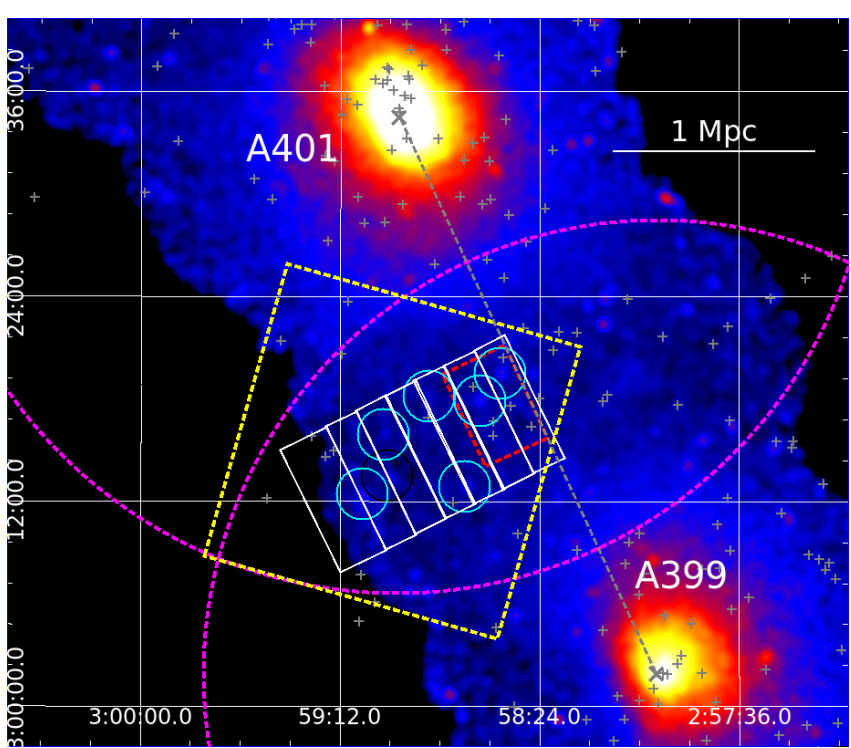

Fig. 1. XMM-Newton image of A399 and A401 in the 0.5-2.0 keV band. The yellow box shows the field of view of the Suzaku XIS. Each region is shown in the figure as a red $\left(6^{\prime} \times 4^{\prime}\right)$ or white box $\left(2^{\prime} \times 8^{\prime}\right.$ or $\left.3^{\prime} \times 8^{\prime}\right)$. Magenta circles indicate the virial radius of each cluster. Small cyan circles show the excluded point sources. Gray plus signs represent galaxies with available redshift between $0.07<z<0.08$ in the NASA/IPAC Extragalactic Database (NED).

analysis presented here. We performed event screening with the cosmic-ray cutoff rigidity COR $2>6 \mathrm{GV}$ to increase the signalto-noise ratio. The total exposure times after data screening is $128.3 \mathrm{ks}$.

\section{Spectral analysis and results}

\subsection{Spectral modeling}

We performed spectral analysis of the Suzaku XIS data to obtain the spatial distribution and physical properties of the plasma in the filament between both clusters. The basic strategy of the fitting is similar to Akamatsu et al. (2016). We used an XMM-Newton archival observation for point source identification (green circles in Fig. 1). We excluded the point sources with a flux $>2 \times 10^{-14} \mathrm{erg} / \mathrm{cm}^{2} / \mathrm{s}$ by a $1.5^{\prime}$ radius to take into account the point spread function (PSF) of the Suzaku XRT (Serlemitsos et al. 2007).

For the spectral analysis, we used the $\operatorname{SPEX}^{1}$ (Kaastra et al. 1996) software version 3.03.00. Each spectrum was binned based on the optimal binning method (Kaastra \& Bleeker 2016). The best-fit parameters were obtained by minimizing the C-stat. A decrease in the low-energy efficiency of the XIS due to contamination of the optical blocking filter was also included in ancillary response files (ARFs). We generated ARFs assuming uniformly extended emission from an observed region with a $20^{\prime}$ radius. We carried out spectral fits to the pulse-height spectrum in each region separately. We used the energy ranges of 0.8-8.0 keV for the both detectors.

The observed spectra consist of three different components such as (1) non-X-ray instrumental background (NXB), (2) X-ray emission from the sky objects and, (3) X-ray emission from the filament plasma. The NXB component was estimated from the dark Earth database using the xisnxbgen program in

\footnotetext{
https://WWW. sron.nl/astrophysics-spex
} 


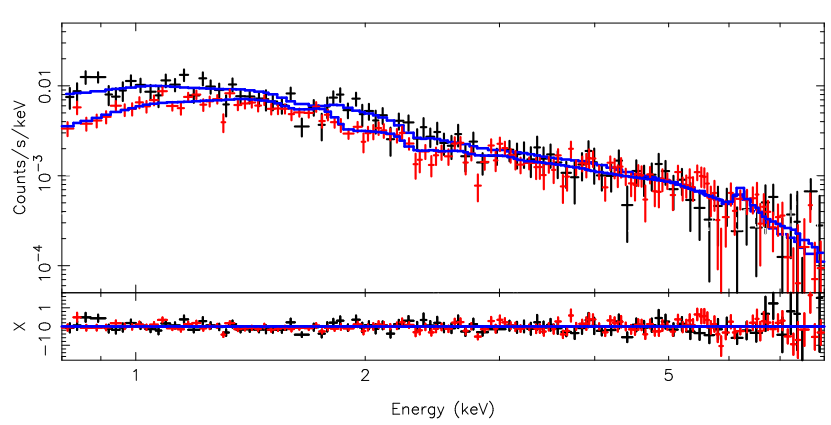

Fig. 2. Example of the spectral fitting. Spectra after subtraction of the NXB and the point sources are shown. The XIS BI (blue) and FI (red) spectra are fitted with CXB + Galactic components (LHB and MWH) and the X-ray emission from the filamentary plasma.

Table 2. Best-fit parameters for the $4^{\prime} \times 6^{\prime}$ box along with the collision axis.

\begin{tabular}{cccc}
\hline \hline $\begin{array}{c}k T \\
(\mathrm{keV})\end{array}$ & $\begin{array}{c}Z \\
\left(Z_{\odot}\right)\end{array}$ & $\begin{array}{c}\text { Norm } \\
\left(10^{69} / \mathrm{m}^{3} / \square^{\prime}\right)\end{array}$ & C-stat/d.o.f. \\
\hline $6.52 \pm 0.35$ & $0.23 \pm 0.08$ & $23.8 \pm 0.5$ & $377 / 299$ \\
\hline
\end{tabular}

FTOOLS (Tawa et al. 2008). To adjust for the long-term variation of the XIS background due to radiation damage, we accumulated the NXB data for the period between 150 days before and 150 days after. The second background component, X-ray emission from sky objects, can be subdivided into Galactic emission and unresolved X-ray sources (cosmic X-ray background; CXB). These background components are modeled during the fitting procedure. We use the following model for these sky background components: cie $+a b s *($ cie + powerlaw). Here, the unabsorbed and absorbed cie components represent X-ray emission from the local hot bubble (LHB) and our Galaxy (the Milky Way halo; MWH), respectively. The power-law component represents the contribution from unresolved point sources $(\mathrm{CXB})$. We fixed the temperature of each thermal component to $0.08 \mathrm{keV}$ and $0.27 \mathrm{keV}$ (Yoshino et al. 2009), respectively. The redshift and abundance of both components were fixed at zero and one solar, respectively. We kept the normalization of thermal components free. For the CXB component, we refer to Kushino et al. (2002).

\subsection{Results}

First, we investigated the properties of filamentary plasma along the collision axis. We extracted the spectra within a $6^{\prime} \times 4^{\prime}(504 \times$ $336 \mathrm{kpc}^{2}$ ) box (red box in Fig. 1) and fitted them with an absorbed thin thermal plasma model ( $a b s \times c i e)$ together with the sky background components discussed above. The best-fit parameters and the spectral fit are shown in Table 2 and Fig. 2, respectively. The top panel of Fig. 3 shows the projected temperature together with previous results (Sakelliou \& Ponman 2004). We also plot a "universa" temperature profile proposed by Burns et al. (2010) as gray dotted curves. The authors performed $N$-body plus hydrodynamic simulations of the cluster formation and compared the density, temperature, and entropy profiles with those of two relaxed clusters observed with Suzaku. From the comparison, they constructed a model of the temperature profile as

$$
\frac{T}{T_{\mathrm{avg}}}=A\left[1+B\left(\frac{r}{r_{200}}\right)\right]^{\beta} \text {. }
$$

The model is in agreement with the two relaxed clusters (Abell 1795 and PKS0745-191) observed with Suzaku and
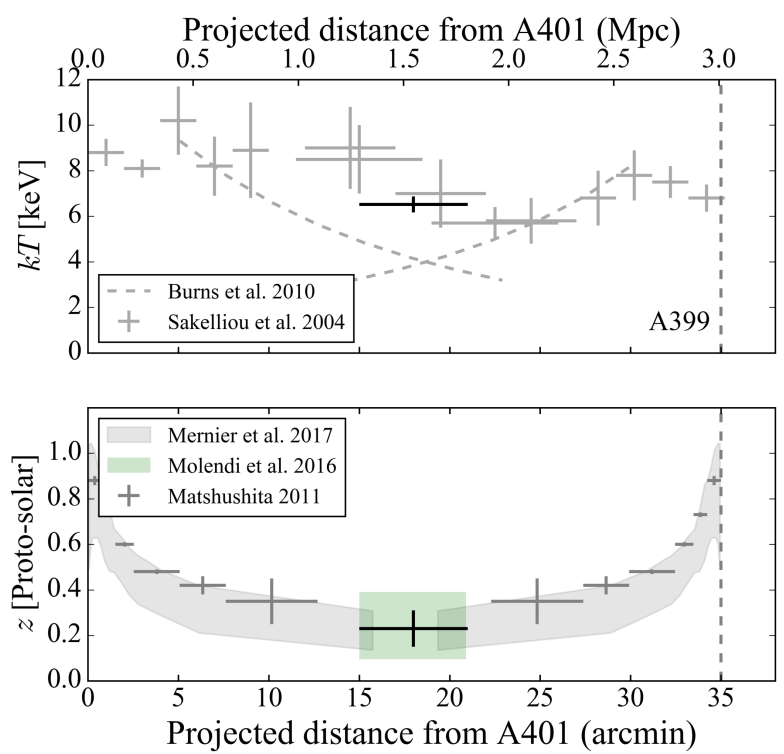

Fig. 3. Top: radial profiles of the temperature of the ICM and filamentary plasma. The Suzaku best-fit value and statistical errors are shown with the black cross. The gray crosses show the previous XMM-Newton measurements by Sakelliou \& Ponman (2004), with converted $1 \sigma$ errors. The gray dashed curves show "universal" temperature profiles (Burns et al. 2010). The gray dashed vertical line shows the approximate position of A399. Bottom: radial profiles of the abundance are shown. All values are converted into Lodders et al. (2009). The gray shaded area (scatter of the sample) and crosses represent the Fe abundance profiles reported by Mernier et al. (2017) and Matsushita (2011), respectively. The green shaded area shows the conservative prediction of the abundance at cluster outskirts by Molendi et al. (2016).

other Suzaku results (see Akamatsu et al. 2011; Reiprich et al. 2013). The temperature estimate of Suzaku significantly exceeds the "universal" profile assuming $T_{\text {avg }}=7.23,8.47 \mathrm{keV}$ (Sakelliou \& Ponman 2004) and $r_{200}=21.9,21.9$ arcmin (Reiprich \& Böhringer 2002) for A399 and A401, respectively. The bottom panel of Fig. 3 shows the abundance profile. We also plot a Fe abundance profiles reported by Mernier et al. (2017) and Matsushita (2011) as the gray shaded area and gray crosses, respectively. Furthermore, our abundance value is in good agreement with the prediction by Molendi et al. (2016), who gave a conservative estimation of the Fe abundance at cluster outskirts.

In order to investigate the spatial distribution of the plasma in the filament, we extracted spectra in 2 or $3 \times 8 \operatorname{arcmin}^{2}$ box regions perpendicular to the collision axis as shown in Fig. 1. We followed the same spectral analysis approach. Intending to measure emission from the space between both clusters, the redshift was fixed to $z=0.073$. We obtained fairly good fits for all the regions with the above model. The best-fit values are summarized in Table 3. The radial profiles of temperature, abundance, and normalization $(Y)$ of the cie component are shown in Fig. 4.

To investigate the robustness of the temperature measurements, we consider possible systematic errors due to the background model. The intensity of the filamentary plasma is much stronger than that of the background components. In that case, the major contribution to the systematic error comes from the fluctuations of the CXB intensity. Here we point out that our results are insensitive to the Galactic background components because their emissions are below the energy range that was used for the spectral fitting. In this paper, we checked the effect of the fluctuation of the $\mathrm{CXB}$ intensity by changing the intensity 

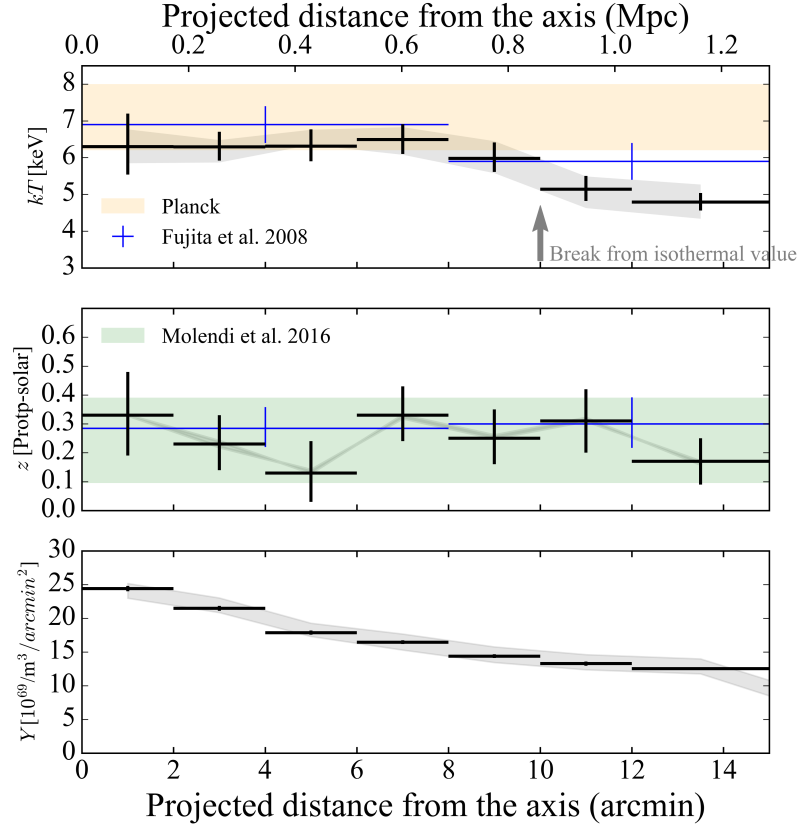

Fig. 4. Top: profiles of the temperature of the filamentary structure as a function of the projected distance from the collision axis shown in Fig. 1. Black crosses indicate the Suzaku best-fit values with statistical errors. The blue crosses show the previous measurements by Fujita et al. (2008), with converted $1 \sigma$ errors. The light orange shaded area represents the temperature estimated by Planck (Planck Collaboration VIII 2013). Middle: radial profiles of the abundance are shown. Colors are same as in the top panel except for the light green shaded area, which shows the best constrained limits of the Fe abundance at cluster outskirts derived by Molendi et al. (2016). Bottom: same as the top panel but for the normalization of the IGM component. The range of uncertainties due to the $30 \%$ fluctuation in the CXB is shown by the gray shaded region.

Table 3. Best-fit parameters of the filamentary plasma.

\begin{tabular}{ccccc}
\hline \hline $\begin{array}{c}\text {Region }^{a} \\
(\operatorname{arcmin})\end{array}$ & $\begin{array}{c}\text { Temperature Abundance } \\
(\mathrm{keV})\end{array}$ & $\begin{array}{c}\text { Norm } \\
\left(\mathrm{Z}_{\odot}\right)\end{array}$ & $\begin{array}{c}\text { C-stat/d.o.f. } \\
\left(10^{69} / \mathrm{m}^{3} / \square^{\prime}\right)\end{array}$ \\
\hline $1.0 \pm 1.0$ & $6.43 \pm 0.87$ & $0.33 \pm 0.15$ & $24.3 \pm 0.6$ & $235.60 / 207$ \\
$3.0 \pm 1.0$ & $6.33 \pm 0.43$ & $0.23 \pm 0.10$ & $21.8 \pm 0.5$ & $302.03 / 233$ \\
$5.0 \pm 1.0$ & $6.37 \pm 0.47$ & $0.13 \pm 0.11$ & $18.4 \pm 0.5$ & $261.96 / 223$ \\
$7.0 \pm 1.0$ & $6.36 \pm 0.41$ & $0.33 \pm 0.10$ & $16.4 \pm 0.4$ & $254.83 / 229$ \\
$9.0 \pm 1.0$ & $5.91 \pm 0.42$ & $0.25 \pm 0.10$ & $14.5 \pm 0.4$ & $278.48 / 231$ \\
$11.0 \pm 1.0$ & $5.07 \pm 0.33$ & $0.31 \pm 0.11$ & $13.3 \pm 0.4$ & $253.28 / 221$ \\
$13.5 \pm 1.5$ & $4.86 \pm 0.27$ & $0.17 \pm 0.08$ & $12.9 \pm 0.3$ & $425.51 / 276$ \\
\hline
\end{tabular}

Notes. ${ }^{(a)}$ Distance from the collision axis.

with $\pm 30 \%$ from the nominal value. In order to estimate the amplitude of the fluctuation, we followed the approach described in Akamatsu et al. (2011). Using the observed area and the flux limit of the excluded point sources, the fluctuations were estimated to be $16-24 \%$. To be conservative, we adopted a fluctuation of $30 \%$ for all regions. The uncertainty due to the CXB fluctuations is shown as the gray shaded area in Fig. 4.

The temperature profile is almost flat at $k T \sim 6-7 \mathrm{keV}$ from the collision axis up to $10^{\prime}(\sim 0.8 \mathrm{Mpc})$, which agrees well with previous results, for example, with XMM-Newton (Sakelliou \& Ponman 2004) and Suzaku (Fujita et al. 2008). In particular, results from Suzaku show excellent agreement with

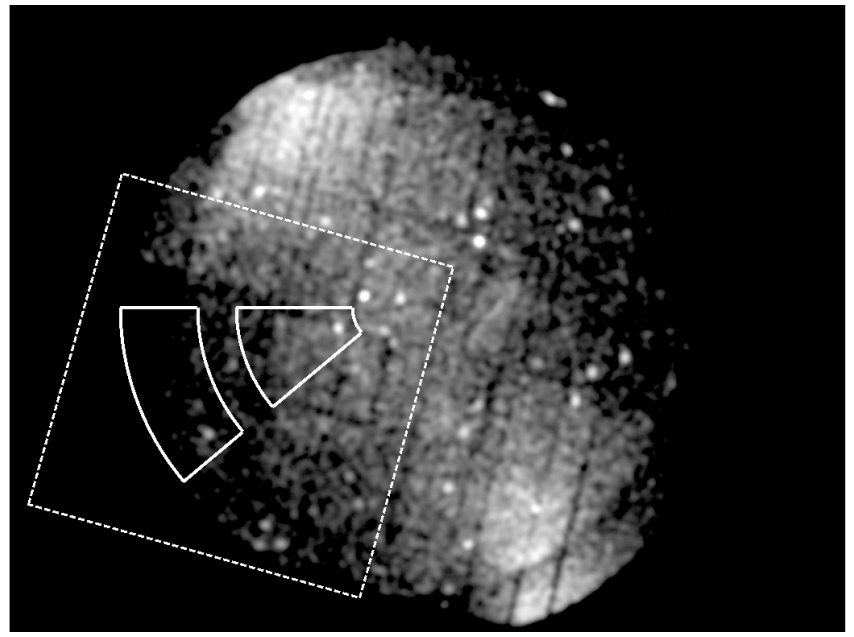

Fig. 5. XMM-Newton image of A399 and A401 in the $0.5-2.0 \mathrm{keV}$ band. The white dotted box shows the field of view of the Suzaku XIS.

Table 4. Best-fit parameters for inside and outside the temperature break.

\begin{tabular}{ccccc}
\hline \hline $\begin{array}{c}\text { Region }^{a} \\
(\operatorname{arcmin})\end{array}$ & $\begin{array}{c}\text { Temperature Abundance } \\
(\mathrm{keV})\end{array}$ & $\begin{array}{c}\text { Norm } \\
\left(Z_{\odot}\right)\end{array}$ & $\begin{array}{c}\text { C-stat/d.o.f. } \\
\left(10^{69} / \mathrm{m}^{3} / \square^{\prime}\right)\end{array}$ \\
\hline $10.0-14.0$ & $5.05 \pm 0.21$ & $0.27 \pm 0.07$ & $13.5 \pm 0.3$ & $488.24 / 346$ \\
$2.0-8.0$ & $6.27 \pm 0.27$ & $0.28 \pm 0.07$ & $18.6 \pm 0.3$ & $369.97 / 309$ \\
\hline
\end{tabular}

Notes. ${ }^{(a)}$ Distance from the collision axis.

the tSZ measurement from Planck $(k T=7.1 \pm 0.9 \mathrm{keV}$ : Planck Collaboration VIII 2013). Across $r=8^{\prime}-10^{\prime}$, the temperature of the filamentary plasma deviates from the isothermal value $k T \sim 6.5 \mathrm{keV}$ to $k T \sim 5 \mathrm{keV}$. To investigate the temperature break, we extracted spectra by selecting annuli from 2.0$8.0^{\prime}$ and 10.0-14.0' from the collision axis with an opening angle of $180^{\circ}-240^{\circ}$ (Fig. 5). The results are summarized in Table 4 and are consistent with the results of the box-shaped analysis. Although the significance is low because of limited statistics, we also found a sign of a break in the X-ray surface brightness profile from a short XMM-Newton observation. We extracted the surface brightness profile from the data and made a model with a broken power-law density profile that includes a jump from a shock using the PROFFIT package (Eckert et al. 2012). The profile and best-fit model are shown in Fig. 6. Considering the rarity of the cold front beyond $0.5 r_{200}$ (see Fig. 5 in Walker et al. 2014), we conclude that the temperature break is a shock front. In the next section, we discuss a possible origin of this temperature break.

The middle panel of Fig. 4 shows the radial profile of the abundance in the filament plasma. Thanks to the low and stable detector background of Suzaku XIS, we were able to derive abundances up to $\sim 1.3 \mathrm{Mpc}(15 \mathrm{arcmin})$ with a reasonable accuracy. The measured abundance values are in good agreement with the previous measurements (after considering the difference of the abundance table: Fujita et al. 2008) and results of the Perseus cluster (Werner et al. 2013).

\section{Discussion}

Suzaku performed a deep observation of the filament plasma between A399 and A401. As a result of low and stable instrumental background and high sensitivity to diffuse sources of the Suzaku XIS, we were able to detect X-ray emission 


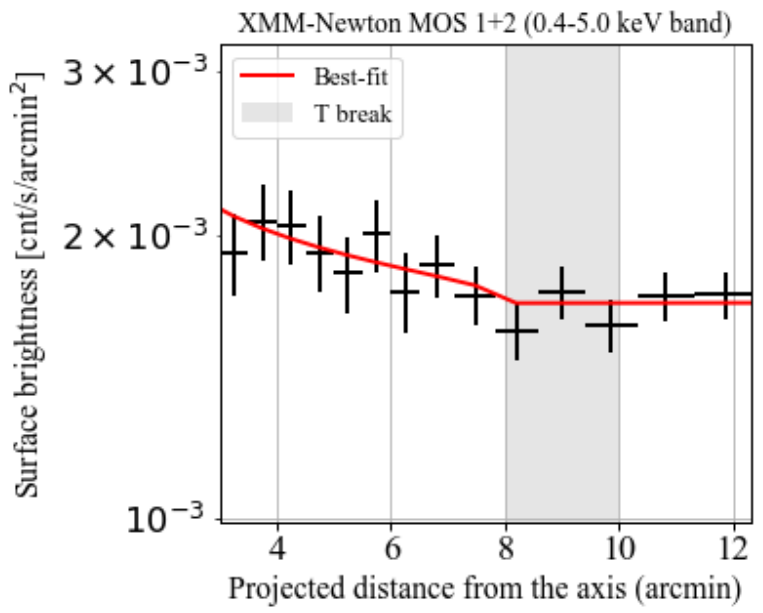

Fig. 6. XMM-Newton surface brightness profile in the $0.4-5.0 \mathrm{keV}$ band. The best-fit profile was derived via the PROFFIT package (Eckert et al. 2012).

up to $1.3 \mathrm{Mpc}$ from the collision axis. In the next subsections, we briefly discuss the implication of these results.

\subsection{Properties and geometry of the filamentary plasma}

First we estimate the properties of the filament plasma. We deduce an electron density in the filament from the CIE normalization. Assuming a volume $V$ of a long-box $\left(1 \operatorname{arcmin}^{2} \times l, l\right.$ is a line-of-sight depth of the volume) and a constant density within the assuming volume, the normalization of the $c$ ie model is $Y=\int n_{\mathrm{e}} n_{\mathrm{H}} V \times 10^{64} \mathrm{~m}^{-3}$, where, $n_{\mathrm{e}} \sim 1.2 n_{\mathrm{H}}$ and $V$ is the volume. Here $n_{\mathrm{H}}$ represents the hydrogen density. From our analysis (Table 3), the electron density in isothermal regions can be estimated as

$n_{\mathrm{e}}=n_{\mathrm{e} 0}\left(\frac{l}{\mathrm{Mpc}}\right)^{-0.5} \mathrm{~cm}^{-3}$,

where $n_{\mathrm{e} 0}=2.5-3.3 \times 10^{-4}$. This value is broadly consistent with the previous estimation $\left(n_{\mathrm{e}} \sim 3.4 \times 10^{-4} \mathrm{~cm}^{-3}\right.$; Fujita et al. 2008). Here, cosmological simulations suggest an exponential density profile perpendicular to the collision axis (see Sect. 1).

The entropy ( $K=k T / n_{\mathrm{e}}^{2 / 3}$ ) of the ICM preserves the heating history of clusters. Therefore the entropy is a nice tracer of cluster growth (Voit 2005). Combined with the measured temperature and electron density, we estimate the entropy of the filament. The estimated entropy is $K \sim 1300 \mathrm{keV} \mathrm{cm}{ }^{2}$. Because of heating by accretion shocks, the entropy of the ICM increases toward the cluster outskirts. Previous XMM-Newton and Chandra observations revealed that the entropy of the ICM well exceeds $K>1000 \mathrm{keV} \mathrm{cm}{ }^{2}$ above $1 \mathrm{Mpc}$ (Pratt et al. 2010; Cavagnolo et al. 2009). Our measurement covers up to the virial radius for each cluster. At the cluster outskirts, the entropy is expected to show a much higher value. The right panel of Fig. 7 shows the radial entropy profile of A399 (red) and A401 (blue), respectively (Cavagnolo et al. 2009). Our measured entropy range is shown by the black cross. The measured low entropy indicates that the filament has not reached hydrodynamic equilibrium. In other words, the filamentary plasma is still not part of the clusters. However, Suzaku observations of clusters revealed that the entropy profiles tend to show lower value than observed from the simulations (e.g., Walker et al. 2012).
To make a firm conclusion, mapping observations are strongly desired.

Another important feature of the filamentary plasma is the report of a tSZ effect signal by Planck (Planck Collaboration VIII 2013). Because of the different dependencies on the temperature and electron density, the combination of the Compton $y$ parameter and X-ray surface brightness allow us to evaluate the electron density and the line of sight length. The $y$ parameter can be described as follows (Sunyaev \& Zeldovich 1972):

$y \equiv \int \frac{k_{\mathrm{B}} T_{\mathrm{e}}}{m_{\mathrm{e}} c^{2}} n_{\mathrm{e}} \sigma_{T} \mathrm{~d} l$

where $k_{\mathrm{B}}$ is the Boltzmann constant, $T_{\mathrm{e}}$ is the electron temperature, $\sigma_{\mathrm{T}}$ is the Thomson cross-section, $n_{\mathrm{e}}$ is the electron number density, $m_{\mathrm{e}} c^{2}$ is the electron rest mass energy, and the integration is along the line of sight. Here, we estimate the Compton $y$-parameter from the Suzaku results. Using $k T=6.5 \pm 0.5 \mathrm{keV}$ and $n_{\mathrm{e}}=3.0 \pm 0.3 \times 10^{-4}(l / \mathrm{Mpc})^{-0.5} \mathrm{~cm}^{-3}$, the estimated $y$-parameter is $14.5 \pm 1.8(l / \mathrm{Mpc})^{0.5} \times 10^{-6}$. Compared with the result of Planck $\left(14-17 \times 10^{-6}\right.$ : see Fig. 2 in Planck Collaboration VIII 2013), the line-of-sight length and the electron density were estimated to be $l=1.1 \mathrm{Mpc}$ and $n_{\mathrm{e}}=$ $3.1 \times 10^{-4} \mathrm{~cm}^{-3}$, respectively. Owing to its stable and low background level, Suzaku successfully detected X-ray emission from the filamentary structure up to $r \sim 15^{\prime}(1.3 \mathrm{Mpc})$ from the connecting axis. Assuming that the other side of the filament also has a similar scale, the filamentary structure may span $2.6 \mathrm{Mpc}$ with a depth of $1.1 \mathrm{Mpc}$. This indicates that the morphology of the filamentary structure is likely a pancake shape rather than a cylindrical shape. The combination of future high-sensitivity SZ and X-ray observations allows us to perform a better three-dimensional tomography of cosmic filaments. Assuming a volume of $2.6 \times 1.1 \times 1.0 \mathrm{Mpc}^{3}$ and a constant density of $n_{\mathrm{e}}=3.1 \times 10^{-4} \mathrm{~cm}^{-3}$, we obtain the gas mass of $M_{\text {gas }} \sim 1.4 \times 10^{13} M_{\odot}$. We stress, however, that the estimated gas mass includes some contributions from both clusters. Furthermore, the assumption of a uniform density is a strong simplification. Therefore, the quoted gas mass has to be considered as an upper limit. Assuming the cosmic baryon fraction of $18 \%$ (Komatsu et al. 2011), the expected total mass of the filament is $7.7 \times 10^{13} M_{\odot}$, which corresponds to about $10 \%$ of the total mass of A399 and A401, respectively. We refer to the total mass of A399 and A401 from Sakelliou \& Ponman (2004) as $5 \times 10^{14} M_{\odot}$ and $6.1 \times 10^{14} M_{\odot}$, respectively.

\subsection{Abundances at the cluster outskirts}

Using the same observation (and, therefore, within the same field of view as shown in Fig. 1), Fujita et al. (2008) measured a significant metallicity value of $\sim 0.2 Z_{\odot}$ in two large bins ( $\sim 8$ arcmin broad each) out to the virial radii of both clusters. They concluded that the presence of these metals at large radii bear witness to powerful galactic superwinds having contributed to enriching the forming ICM at high redshift, i.e., before the clusters started to assemble. Since Fe is predominantly produced by Type Ia supernovae (SNIa; e.g., de Plaa et al. 2007; Mernier et al. 2016), it is clear that a significant fraction of SNIa that contributed to the ICM enrichment must have exploded early on, i.e., before or when those galactic superwinds occurred. A similar result, also interpreted as the signature of an early enrichment by SNIa, has been found in the outskirts of the Perseus cluster (Werner et al. 2013). This also suggests that the positive $\mathrm{Fe}$ abundance previously found in the outskirts of A 399-A 401 

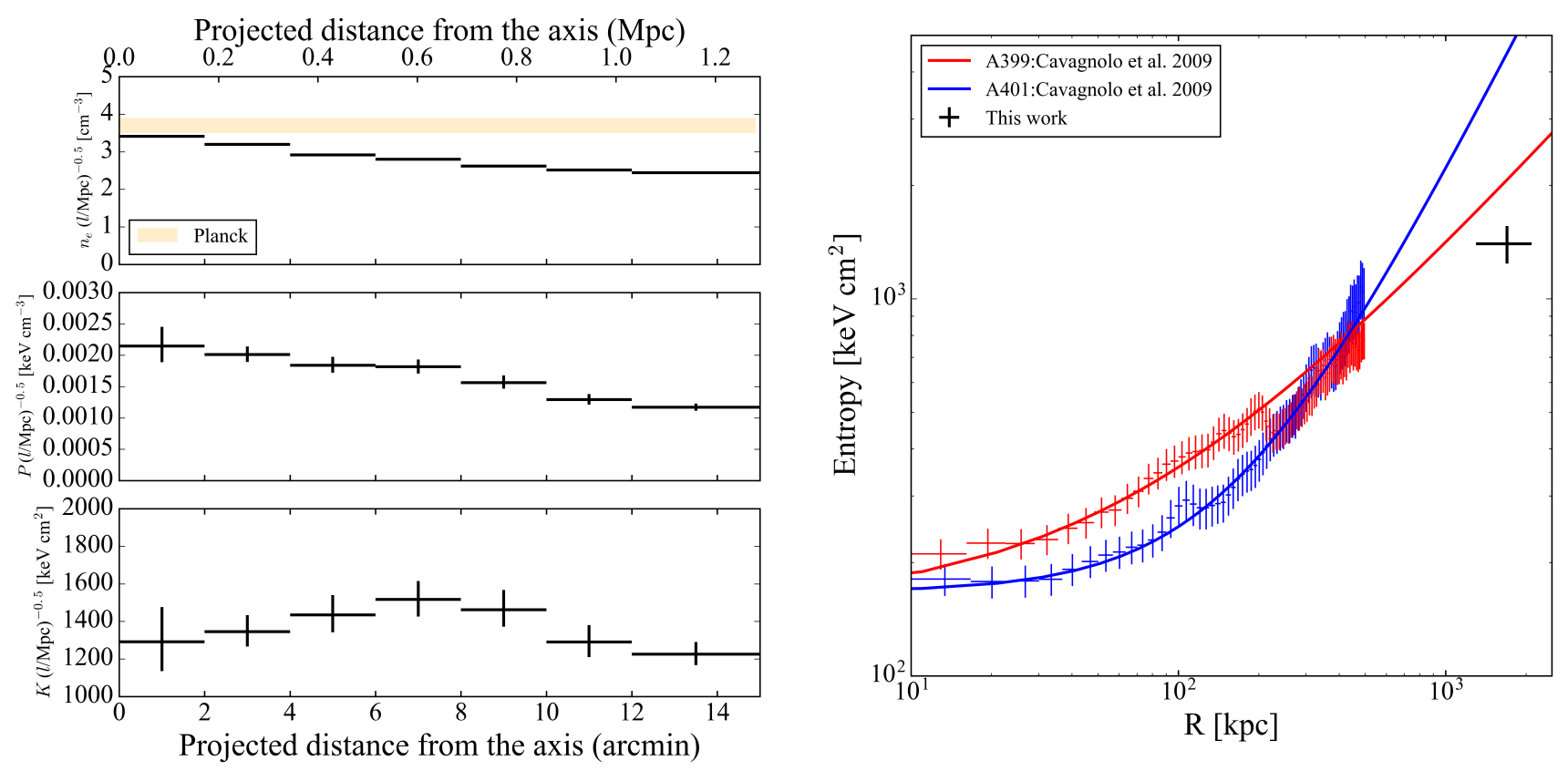

Fig. 7. Left: radial profiles of the pseudo-electron density, thermal pressure, and entropy. In the top panel, the orange region indicates the electron density from the Planck result of Planck Collaboration VIII (2013). Right: radial entropy profiles for A399 (red) and A401, respectively. Crosses show the results from Chandra observations (Cavagnolo et al. 2009). The solid line represents the empirical model $K(r)=K_{0}+K_{100}(r / 100 \mathrm{kpc})^{\alpha}$. We adopt the best-fit parameters from (Cavagnolo et al. 2009), i.e., $K_{0: A 399}=153.20, K_{100: A 399}=204.34, \alpha_{A 399}=0.79, K_{0: A 401}=166.80, K_{100: A 401}=$ $81.79, \alpha_{A 401}=1.40$, respectively.

is likely not related to the particular configuration of these two systems (pre-merging phase).

However, the two measurements alone in the analysis of Fujita et al. (2008) left some remaining doubt regarding the uniformity of the Fe abundance. From our reanalysis, and as shown by Fig. 4 (middle panel), it clearly appears that the Fe distribution remains remarkably uniform at smaller scales $(\sim 200 \mathrm{kpc})$ and within error bars.

That said, care must be taken when interpreting metallicities in cluster outskirts. Even assuming (very optimistically) that the NXB and CXB components affecting spectra of low signaltonoise regions are perfectly understood and under control, the moderate PSF of the XIS instrument prevents us from checking the spatial uniformity of metals below $200 \mathrm{kpc}$. In particular, Molendi et al. (2016) showed that, using current CCD spectrometers, the presence of unresolved cooler, denser, and more metal-rich substructures in the outskirts would significantly bias high the actual mass-weighted metallicity (typically by a factor of $\sim 3$ ). Similarly, the limited quality of our spectra does not allow us to probe the thermal structure of the ICM at such a large distance from the core. Since the equivalent widths of the metal lines strongly depend on the plasma temperature, an inappropriate single-temperature modeling would affect the abundance measurements in case of a non-isothermal ICM (see, e.g., Fujita et al. 1996a, 1997; Buote 2000)

Keeping these uncertainties in mind, it must be noted (Fig. 3, bottom) that our average Fe abundance measurement of $\sim 0.2 Z_{\odot}$ (thus consistent with the value of Fujita et al. 2008) agrees very well with the extrapolation of the radial $\mathrm{Fe}$ abundance profiles in clusters derived by Matsushita (2011) and Mernier et al. (2017). Assuming, as suggested above, that the Fe content of our investigated region is independent of the actual merging state of A 399-A 401, our results give one more hint of (at least) two distinct steps in the ICM enrichment history: first, an early enrichment, presumably via galactic superwinds before the cluster assembly, responsible for a uniform Fe floor (see above); and, second, in case of cool-core clusters, a later enrichment, likely due to the central cD galaxy, responsible for the central Fe peak ${ }^{2}$.

Besides $\mathrm{Fe}$, the $\mathrm{O}, \mathrm{Mg}, \mathrm{Si}$, and $\mathrm{S}$ abundances can in principle be measured with Suzaku (e.g., Sato et al. 2007). Since these four elements are synthesized (at least partly) by core-collapse supernovae ( $\mathrm{SNcc}$ ), measuring their abundances at large radii can provide us valuable information on the role of SNcc in the ICM enrichment history (e.g., Simionescu et al. 2015; Ezer et al. 2017). Unfortunately, the limited exposure of our data, together with the moderate spectral resolution of XIS and the high temperature plasma giving rise to weaker metal line emissivities, prevent us from constraining the $\mathrm{O}, \mathrm{Mg}, \mathrm{Si}$ and $\mathrm{S}$ abundances in the region studied here. Future missions will be particularly useful to tackle this issue.

\subsection{Edge and origin of the filamentary hot plasma}

The high sensitivity and low background of Suzaku enable us to estimate the temperature structure of the filament with a high accuracy. In the region between both clusters, we find a clear enhancement in the temperature profile of ICM from $k T \sim 4 \mathrm{keV}$ (expected from the universal profile) to $\sim 6.5 \mathrm{keV}$ (Fig. 3). This indicates that the clusters have been interacting even though the accrued distance between the clusters $(\sim 7 \mathrm{Mpc}$ assuming the redshift difference between the clusters is only due to the Hubble flow) is larger than their virial radii $(\sim 2 \mathrm{Mpc})$.

2 In principle, ram-pressure stripping of infalling cluster galaxies could also produce a centrally peaked Fe distribution (Domainko et al. 2006); however its predicted extent $(\sim 1 \mathrm{Mpc})$ is considerably broader than what is actually observed (e.g., Simionescu et al. 2009). 

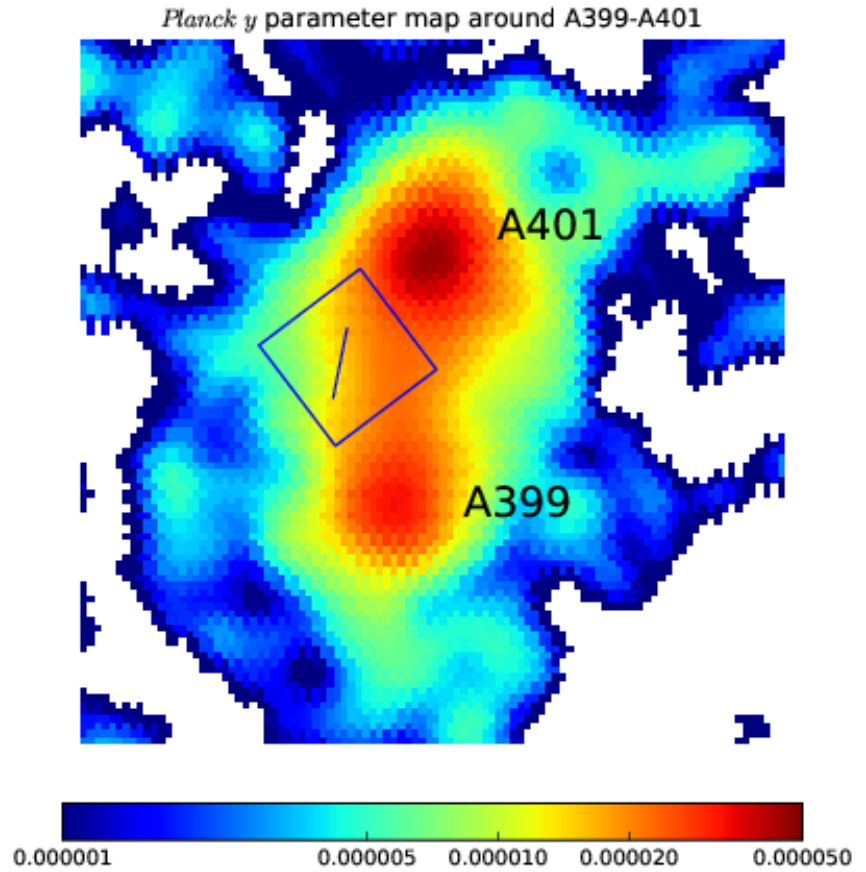

Fig. 8. Compton $y$ parameter around A399-A401 from Planck Collaboration XXII (2016). Blue box and line represent the Suzaku FOV and the location of the temperature break from the isothermal region.

Similar temperature enhancements were also reported in the A1750, Cygnus A, CIZA J1358.9-4750, and 1E2216.0-0401 system (Belsole et al. 2004; Molnar et al. 2013; Sarazin et al. 2013; Kato et al. 2015; Akamatsu et al. 2016). For all those cases each of the two clusters is partially located within the virial radius of its respective companion. That is, it is natural to think that the cluster pair already starts interacting and most probably the temperature enhancement is caused by a compression induced by the interaction. On the other hand, in the A3528 case, there is no clear evidence of the enhancement in both the temperature and surface brightness (Gastaldello et al. 2003). A similar result has also been reported in the A3556 \& A3558 system (Mitsuishi et al. 2012). That is, in the case where the separation of the pair is much larger than their virial radius, the temperature enhancement is a not so common phenomena.

Our scenario is that A399\&A401 originally had a filament connecting the clusters, which caused an accretion flow onto the filament and formed a shock front parallel to the connecting axis. During the evolution of the clusters, the filament is (adiabatically) compressed by the merging activity. This increases the gravitational force of the filament. Consequently, the filament should have a strong accretion flow onto it. In this case, the observed filament plasma could not be the ICM of the clusters only, most likely it is a mixture of the ICM of the clusters and IGM, which was not captured by the clusters yet. As we discuss in the next section (Sect. 4.4), predictions of cosmological hydrodynamical simulations (Dolag et al. 2006) of a filament located between two clusters match these measured properties of our studied filament candidate, such as uniform temperature, $y$ parameter within the filament, and a possible shock front parallel to the connecting axis. Furthermore, we investigated a relationship between the $y$ parameter measured by Planck and the observed temperature break. The bottom panel of Fig. 8 shows the $y$-parameter map reported by the Planck Collaboration XXII (2016).
Based on the assumption that the temperature break is a shock front, we briefly discuss the implication of these results. To evaluate the intensity of the shock, the Mach number, we applied the Rankine-Hugoniot jump condition,

$\frac{T_{2}}{T_{1}}=\frac{5 \mathcal{M}^{4}+14 \mathcal{M}^{2}-3}{16 \mathcal{M}^{2}}$,

where subscripts 1 and 2 denote pre-shock and post-shock values, respectively. Here, we assume the post- and pre-shock temperature as $k T_{\text {post }} \sim 6.3 \mathrm{keV}, k T_{\text {pre }} \sim 5.1 \mathrm{keV}$ (Table 4). The resultant Mach number is about $\mathcal{M} \sim 1.3$. These results support the interpretation that the shock that we are witnessing is associated with the rim of the filament, which was formed by the accretion flow due to the gravitational force by the filament.

Comparing these results with recent findings of a shock front in an early phase merging cluster (Kato et al. 2015; Akamatsu et al. 2016), the property (the Mach number) of the shocks is almost consistent with each other. The location of these shocks are well matched with the prediction of numerical simulations of major merger events (Takizawa 1999; Ricker \& Sarazin 2001). Contrary to the other samples, we observed the shock front at a different geometry. Previous hydrodynamical simulations of A399 and A401 without the contribution of the filament (Akahori \& Yoshikawa 2008) also failed to predict such a shock front with a pure binary cluster merger. XMM-Newton observations revealed that the surface brightness profile between A399 and A401 cannot be explained by just simple superposition of the two clusters (see the right panel of Fig. 10 in Sakelliou \& Ponman 2004). Furthermore, our work suggests there is more mass than the simple superposition. These observational facts support the presence of a considerable amount of WHIM filament between the clusters at initial phase.

\subsection{Comparison with the hydrodynamical simulations}

The timescale of the evolution of such large-scale filaments and clusters of galaxies is compatible with the Hubble time. In this case, there are two approaches to understanding their full evolution history. The first approach is making snapshots of the history by collecting several samples that are at a different evolution phase. Another approach is a comparison with numerical simulations, which is able to track the full evolution of such large-scale structures with already known physics.

In practice, the number of observed cases is not very large. Thus, we compare our results with previously performed numerical simulations. We summarized our results as follows:

1. A nearly isothermal $(k T \sim 6-7 \mathrm{keV})$ filament between two clusters of galaxies: Sect. 4.1 and Fig. 4.

2. The Compton $y$ parameter of the tSZ effect is $\sim 1.5 \times 10^{-5}$ : Sect. 4.3 and Fig. 8

3. The possible presence of a (weak) shock at the outer part of the filament: Sect. 4.3 and Fig. 4.

We compare these results with the simulations presented in Dolag et al. (2006). The simulations concentrate on describing the physical properties of a supercluster-like region including the treatment of radiative cooling, heating by a uniform UV background and star formation feedback processes. For the comparison, we selected the cluster pair named as halo-b and halo-d (see Table 1 for the details). This cluster pair reasonably mimics the situation of A399-A401. At $z=0.07$, the physical separation of the pair is about $4 \mathrm{Mpc} / h(3.0 \mathrm{Mpc} / h$ in the projection on the 
sky plane). The main features of halo b-d can be summarized as follows:

1. Accretion flows onto the filament, which generates shock fronts parallel to the axis joining the two haloes; Sect. 3 in Dolag et al.

2. Owing to the outward movement of the accretion, shocks are created and extended, almost to isothermal regions $(k T>$ $4.3 \mathrm{keV}$ ); Sect. 4 and the middle of the left panel of Fig. 6

3. The tSZ Compton $y$ parameter can be on the order of $\sim 10^{-5}$ : Fig. 10.

These physical properties of cosmic filaments predicted by the simulation (Dolag et al. 2006) are, qualitative speaking, in excellent agreement with our measurements of the filamentary plasma between A399-A401. Therefore, the observed temperature break is likely the shock front related to the structure formation, which is still propagating outward together with ongoing evolution of the filament.

Acknowledgements. The authors thank the referee for constructive comments which have helped to improve the paper. The authors would like to thank all the members of the Suzaku team for their continuous contributions in the maintenance of onboard instruments, calibrations, and software development. H.A acknowledges the support of NWO via a Veni grant. Y.F. acknowledges financial support from MEXT KAKENHI No. 15K05080. SRON is supported financially by NWO, The Netherlands Organization for Scientific Research.

\section{References}

Akahori, T., \& Yoshikawa, K. 2008, PASJ, 60, L19

Akamatsu, H., Hoshino, A., Ishisaki, Y., et al. 2011, PASJ, 63, 1019 Akamatsu, H., Gu, L., Shimwell, T. W., et al. 2016, A\&A, 593, L7

Anders, E., \& Grevesse, N. 1989, Geochim. Cosmochim. Acta, 53, 197

Belsole, E., Pratt, G. W., Sauvageot, J.-L., \& Bourdin, H. 2004, A\&A, 415, 821

Buote, D. A. 2000, MNRAS, 311, 176

Buote, D. A., Zappacosta, L., Fang, T., et al. 2009, ApJ, 695, 1351

Burns, J. O., Skillman, S. W., \& O'Shea, B. W. 2010, ApJ, 721, 1105

Cavagnolo, K. W., Donahue, M., Voit, G. M., \& Sun, M. 2009, ApJS, 182, 12

Cen, R., \& Ostriker, J. P. 1999, ApJ, 514, 1

Colberg, J. M., Krughoff, K. S., \& Connolly, A. J. 2005, MNRAS, 359, 272

Davé, R., Cen, R., Ostriker, J. P., et al. 2001, ApJ, 552, 473

de Plaa, J., Werner, N., Bleeker, J. A. M., et al. 2007, A\&A, 465, 345

Dickey, J. M., \& Lockman, F. J. 1990, ARA\&A, 28, 215

Dolag, K., Meneghetti, M., Moscardini, L., Rasia, E., \& Bonaldi, A. 2006, MNRAS, 370, 656

Domainko, W., Mair, M., Kapferer, W., et al. 2006, A\&A, 452, 795

Eckert, D., Vazza, F., Ettori, S., et al. 2012, A\&A, 541, A57

Eckert, D., Jauzac, M., Shan, H., et al. 2015, Nature, 528, 105

Ezer, C., Bulbul, E., Nihal Ercan, E., et al. 2017, ApJ, 836, 110

Fang, T., Buote, D. A., Humphrey, P. J., et al. 2010, ApJ, 714, 1715

Fujimoto, R., Takei, Y., Tamura, T., et al. 2004, PASJ, 56, L29

Fujita, Y., Fukumoto, J., \& Okoshi, K. 1996a, ApJ, 470, 762

Fujita, Y., Koyama, K., Tsuru, T., \& Matsumoto, H. 1996b, PASJ, 48, 191

Fujita, Y., Fukumoto, J., \& Okoshi, K. 1997, ApJ, 488, 585
Fujita, Y., Tawa, N., Hayashida, K., et al. 2008, PASJ, 60, S343 Fukugita, M., Hogan, C. J., \& Peebles, P. J. E. 1998, ApJ, 503, 518 Galeazzi, M., Gupta, A., \& Ursino, E. 2009, ApJ, 695, 1127 Gastaldello, F., Ettori, S., Molendi, S., et al. 2003, A\&A, 411, 21 Kaastra, J. S., \& Bleeker, J. A. M. 2016, A\&A, 587, A151

Kaastra, J. S., Mewe, R., \& Nieuwenhuijzen, H. 1996, in UV and X-ray Spectroscopy of Astrophysical and Laboratory Plasmas, eds. K. Yamashita, \& T. Watanabe, 411

Kaastra, J. S., Werner, N., Herder, J. W. A. D., et al. 2006, ApJ, 652, 189

Kang, H., Ryu, D., \& Jones, T. W. 1996, ApJ, 456, 422

Kato, Y., Nakazawa, K., Gu, L., et al. 2015, PASJ, 67, 71

Komatsu, E., Smith, K. M., Dunkley, J., et al. 2011, ApJS, 192, 18

Koyama, K., Tsunemi, H., Dotani, T., et al. 2007, PASJ, 59, 23

Kushino, A., Ishisaki, Y., Morita, U., et al. 2002, PASJ, 54, 327

Lodders, K., Palme, H., \& Gail, H.-P. 2009, Landolt Börnstein [arXiv:0901.1149]

Matsushita, K. 2011, A\&A, 527, A134

Mernier, F., de Plaa, J., Pinto, C., et al. 2016, A\&A, 595, A126

Mernier, F., de Plaa, J., Kaastra, J. S., et al. 2017, A\&A, 603, A80

Miniati, F. 2003, MNRAS, 342, 1009

Miniati, F., Jones, T. W., Kang, H., \& Ryu, D. 2001a, ApJ, 562, 233

Miniati, F., Ryu, D., Kang, H., \& Jones, T. W. 2001b, ApJ, 559, 59

Mitsuishi, I., Gupta, A., Yamasaki, N. Y., et al. 2012, PASJ, 64, 18

Molendi, S., Eckert, D., De Grandi, S., et al. 2016, A\&A, 586, A32

Molnar, S. M., Chiu, I.-N. T., Broadhurst, T., \& Stadel, J. G. 2013, ApJ, 779, 63

Nicastro, F., Mathur, S., Elvis, M., et al. 2005, Nature, 433, 495

Nicastro, F., Elvis, M., Krongold, Y., et al. 2013, ApJ, 769, 90

Oegerle, W. R., \& Hill, J. M. 2001, AJ, 122, 2858

Planck Collaboration VIII. 2013, A\&A, 550, A134

Planck Collaboration XXII. 2016, A\&A, 594, A22

Pratt, G. W., Arnaud, M., Piffaretti, R., et al. 2010, A\&A, 511, A85

Reiprich, T. H., \& Böhringer, H. 2002, ApJ, 567, 716

Reiprich, T. H., Basu, K., Ettori, S., et al. 2013, Space Sci. Rev., 177, 195

Ricker, P. M., \& Sarazin, C. L. 2001, ApJ, 561, 621

Ryu, D., Kang, H., Hallman, E., \& Jones, T. W. 2003, ApJ, 593, 599

Sakelliou, I., \& Ponman, T. J. 2004, MNRAS, 351, 1439

Sarazin, C. L., Finoguenov, A., \& Wik, D. R. 2013, Astron. Nachr., 334, 346

Sato, K., Tokoi, K., Matsushita, K., et al. 2007, ApJ, 667, L41

Serlemitsos, P. J., Soong, Y., Chan, K.-W., et al. 2007, PASJ, 59, 9

Simionescu, A., Werner, N., Böhringer, H., et al. 2009, A\&A, 493, 409

Simionescu, A., Werner, N., Urban, O., et al. 2015, ApJ, 811, L25

Skillman, S. W., O'Shea, B. W., Hallman, E. J., Burns, J. O., \& Norman, M. L. 2008, ApJ, 689, 1063

Springel, V., White, S. D. M., Jenkins, A., et al. 2005, Nature, 435, 629

Sunyaev, R. A., \& Zeldovich, Y. B. 1972, Comm. Astrophys. Space Phys., 4, 173

Takei, Y., Fujimoto, R., Mitsuda, K., \& Onaka, T. 2002, ApJ, 581, 307

Takizawa, M. 1999, ApJ, 520, 514

Tawa, N., Hayashida, K., Nagai, M., et al. 2008, PASJ, 60, 11

Ulmer, M. P., \& Cruddace, R. G. 1981, ApJ, 246, L99

Ulmer, M. P., Kinzer, R., Cruddace, R. G., et al. 1979, ApJ, 227, L73

Voit, G. M. 2005, Rev. Mod. Phys., 77, 207

Walker, S. A., Fabian, A. C., Sanders, J. S., \& George, M. R. 2012, MNRAS, 427, L45

Werner, N., Finoguenov, A., Kaastra, J. S., et al. 2008, A\&A, 482, L29

Werner, N., Urban, O., Simionescu, A., \& Allen, S. W. 2013, Nature, 502, 656

Yoshikawa, K., Yamasaki, N. Y., Suto, Y., et al. 2003, PASJ, 55, 879

Yoshikawa, K., Dolag, K., Suto, Y., et al. 2004, PASJ, 56, 939

Yoshino, T., Mitsuda, K., Yamasaki, N. Y., et al. 2009, PASJ, 61, 805

Zappacosta, L., Nicastro, F., Maiolino, R., et al. 2010, ApJ, 717, 74 